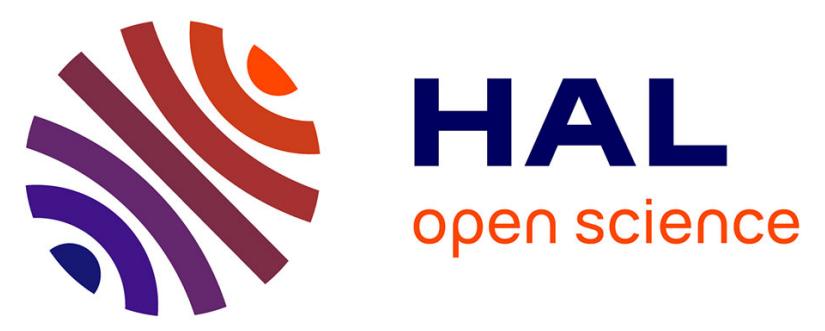

\title{
Structural analysis of a glycoside hydrolase family 43 arabinoxylan arabinofuranohydrolase in complex with xylotetraose reveals a different binding mechanism compared to other members of the same family
}

Elien Vandermarliere, Tine M Bourgois, Martyn D. Winn, Steven van Campenhout, Guido Volckaert, Jan A. Delcour, Sergei V. Strelkov, Anja Rabijns, Christophe M. Courtin

\section{- To cite this version:}

Elien Vandermarliere, Tine M Bourgois, Martyn D. Winn, Steven van Campenhout, Guido Volckaert, et al.. Structural analysis of a glycoside hydrolase family 43 arabinoxylan arabinofuranohydrolase in complex with xylotetraose reveals a different binding mechanism compared to other members of the same family. Biochemical Journal, 2009, 418 (1), pp.39-47. 10.1042/BJ20081256 . hal-00479054

\section{HAL Id: hal-00479054 https://hal.science/hal-00479054}

Submitted on 30 Apr 2010

HAL is a multi-disciplinary open access archive for the deposit and dissemination of scientific research documents, whether they are published or not. The documents may come from teaching and research institutions in France or abroad, or from public or private research centers.
L'archive ouverte pluridisciplinaire HAL, est destinée au dépôt et à la diffusion de documents scientifiques de niveau recherche, publiés ou non, émanant des établissements d'enseignement et de recherche français ou étrangers, des laboratoires publics ou privés. 
Structural analysis of a glycoside hydrolase family 43 arabinoxylan arabinofuranohydrolase in complex with xylotetraose reveals a different binding mechanism compared to other members of the same family.

Elien Vandermarliere*, Tine M. Bourgois ${ }^{\dagger}$, Martyn D. Winn*, Steven Van Campenhout ${ }^{\dagger}$, Guido Volckaert $^{\dagger}$, Jan A. Delcour ${ }^{\S}$, Sergei V. Strelkov ${ }^{*}$, Anja Rabijns ${ }^{*}$, Christophe M. Courtin ${ }^{\S}$

*Laboratory for Biocrystallography, Department of Pharmaceutical Sciences, Katholieke Universiteit Leuven, Herestraat 49, O\&N II, bus 822, 3000 Leuven, Belgium

${ }^{\dagger}$ Laboratory of Gene Technology, Department of Biosystems, Katholieke Universiteit Leuven, Kasteelpark Arenberg 21, bus 2462, 3001 Leuven, Belgium

*Computational Science and Engineering Department, STFC Daresbury Laboratory, Daresbury, Warrington WA4 4AD, United Kingdom

${ }^{\S}$ Laboratory of Food Chemistry and Biochemistry, Department of Microbial and Molecular Systems, Katholieke Universiteit Leuven, Kasteelpark Arenberg 20, bus 2463, 3001 Leuven, Belgium

Corresponding author: Christophe M. Courtin, Laboratory of Food Chemistry and Biochemistry, Department of Microbial and Molecular Systems, Katholieke Universiteit Leuven, Kasteelpark Arenberg 20, bus 2463, 3001 Leuven, Belgium Tel: +32(0)16321917, Fax: +32(0)16321997 Email: Christophe.Courtin@,biw.kuleuven.be

Running title: The structure of $B$. subtilis GH43 arabinoxylan arabinofuranohydrolase 


\section{Synopsis}

Arabinoxylan arabinofuranohydrolases $(\mathrm{AXH})$ are $\alpha$-L-arabinofuranosidases that specifically hydrolyze the glycosidic bond between arabinofuranosyl substituents and xylopyranosyl backbone residues of arabinoxylan. Bacillus subtilis was recently shown to produce an AXH that cleaves arabinose units from $O-2$ - or $O-3$-monosubstituted xylose residues: BSAXH-m2,3. Crystallographic analysis reveals a two domain structure for this enzyme: a catalytic domain displaying a 5-bladed $\beta$-propeller fold characteristic to glycoside hydrolase family (GH) 43 and a carbohydrate binding module (CBM) with a $\beta$-sandwich fold belonging to CBM family 6 . Binding of substrate to $B S \mathrm{AXH}-\mathrm{m} 2,3$ is largely based on hydrophobic stacking interactions which probably allow the positional flexibility needed to hydrolyze both arabinose substituents at the $O-2$ or $O-3$ position of the xylose unit. Superposition of the $B s$ AXH-m2,3 structure with known structures of the GH family 43 exo-acting enzymes, $\beta$-xylosidase and $\alpha$-L-arabinanase, each in complex with their substrate, reveals a different orientation of the sugar backbone.

Key words: Family 43 glycoside hydrolases, arabinoxylan arabinofuranohydrolase, Bacillus subtilis, enzyme-substrate complexes, substrate binding mechanism, crystallography 


\section{Introduction}

More and more, renewable sources are being researched as an alternative for petroleum-derived fuels and materials. At the moment, the most common renewable fuel is ethanol derived from cereal starch. Because of limitations in cereal supply, a better utilization of the whole kernel is being explored with focus on hemicellulose $[1,2]$. An important hemicellulose is arabinoxylan which is composed of a linear backbone of $\beta$-1,4-linked D-xylopyranosyl units which can be $O-2$ and/or $O-3$ substituted with L-arabinofuranosyl units. Enzymatic degradation of arabinoxylan into its building blocks requires a set of xylanolytic enzymes. Endoxylanases and $\beta$-xylosidases hydrolyze the xylan backbone and act in synergy with arabinofuranosidases which remove the arabinofuranosyl units [3]. These enzymes are thus important players in the conversion of heteroxylan into xylose and arabinose which can then be used either for the production of ethanol or as platform molecules.

$\alpha$-L-Arabinofuranosidases (E.C. 3.2.1.55) are classified according to their substrate specificity. Type A arabinofuranosidases are only active against small substrates such as 4-nitrophenyl $\alpha$-Larabinofuranoside and short-chain arabinoxylo-oligosaccharides (AXOS). Type B arabinofuranosidases can also hydrolyze polymeric substrates such as branched arabinan and arabinoxylan [4]. Some type B arabinofuranosidases specifically cleave arabinofuranosyl units from arabinoxylan and hence are termed arabinoxylan arabinofuranohydrolase (AXH) [5]. The latter type can further be divided in two and possibly three groups. AXH-m release arabinose only from mono-substituted xylose units, while AXH-d release it only from double-substituted xylose units $[6,7]$. Ferré and coworkers suggested that AXH from barley malt releases arabinose from both single- and double-substituted xylose units and therefore, can be classified as AXH$\mathrm{md}[8]$. 
Based on sequence similarity, the currently known arabinofuranosidases have been classified into 5 glycoside hydrolase $(\mathrm{GH})$ families. Those are found in $\mathrm{GH}$ families 3, 51 and 54 hydrolyze the glycosidic bond with retention of the anomeric configuration. Those in GH families 43 and 62 invert the anomeric configuration upon hydrolysis $[9,10]$. To date, the only known structures of arabinofuranosidases are those of arabinofuranosidases belonging to GH families 51 and 54, displaying a $(\beta / \alpha)_{8}$-barrel and a $\beta$-sandwich, respectively $[11,12]$.

Recently, Bourgois and coworkers have characterized XynD from Bacillus subtilis subspecies subtilis ATCC 6051, which was previously predicted to be a member of GH family 43 displaying endoxylanase activity supplemented with arabinofuranosidase co-activity. Careful biochemical analysis characterized it as an arabinoxylan arabinofuranohydrolase that cleaves arabinose units from $O$-2- or $O-3$-monosubstituted xylose residues, i.e. as an AXH-m2,3 [13]. Within GH family 43, structures of $\beta$-xylosidases from Geobacillus stearothermophilus [14], Bacillus halodurans, Bacillus subtilis and Clostridium acetobutylicum are available. Also $\alpha$-L-arabinanases from Cellvibrio japonicus [15] and Bacillus thermodenitrificans [16] and a bifunctional $\beta$ xylosidase/ $\alpha$-L-arabinofuranosidase from Selenomonas ruminantium [17] belonging to $\mathrm{GH}$ family 43 had their structure determined. Together with $\mathrm{GH}$ families 32, 62 and 68, their catalytic domain shares a 5-bladed $\beta$-propeller fold which was first seen for tachylectin [18]. Unlike the classical organization of the active site of inverting enzymes, which consists of a proton donor, the general acid, and a nucleophile, the general base, a third carboxylate was found to be essential for activity in GH family 43 enzymes. This residue is responsible for the $\mathrm{p} K_{\mathrm{a}}$ modulation of the general acid and correct orientation of both the proton donor and substrate [14]. Although the resolved $\alpha$-L-arabinanase structures show no associated carbohydrate binding module (CBM), other GH family 43 members contain a CBM. For B. subtilis AXH-m2,3 
(BsAXH-m2,3) it has been proposed, based on sequence similarity, that this CBM belongs to CBM family 6 [13]. Members of this family display a $\beta$-sandwich fold: a lectin-like $\beta$-jelly roll consisting of 5 antiparallel $\beta$-strands packed against 4 antiparallel $\beta$-strands. Like other CBMs, their function is to increase the effective concentration of the active site on polymeric substrate $[19,20]$.

Since $\beta$-xylosidases, $\alpha$-L-arabinanases and bifunctional $\beta$-xylosidases $/ \alpha$-L-arabinofuranosidases also belong to $\mathrm{GH}$ family 43, they not only have their structure in common, but also an analogous reaction mechanism as their catalytic residues occur at an analogous position [10]. However, unlike $\beta$-xylosidases and $\alpha$-L-arabinanases which are exo-acting enzymes, AXH-m2,3 releases the arabinose substituents of arabinoxylan, so we can expect differences in the substrate binding mechanism. To understand the binding of substrate, knowledge of the three-dimensional structure is essential. For $\beta$-xylosidase and $\alpha$-L-arabinanase, the structure in complex with xylobiose [14] and the structure in complex with arabinohexaose [15], respectively, are available and will be used here as reference structures. No structure of an AXH-m2,3 is available. We have previously reported the crystallization and preliminary X-ray analysis of $B s \mathrm{AXH}-\mathrm{m} 2,3$ in complex with xylotriose [21]. Here, we describe the crystal structure of BsAXH-m2,3, the first structure of a GH family 43 arabinofuranosidase, and the first structure of an AXH overall, in complex with several substrates. The structure allows in depth comparison of the substrate binding mechanism between the GH family 43 classes. 


\section{Experimental}

\section{Crystallization and data collection}

Recombinant AXH-m2,3 from B. subtilis was expressed and purified as described by Bourgois and coworkers [13]. Crystallization and data collection of BsAXH-m2,3 crystals soaked with xylotriose was reported earlier [21]. In short, thick needle-like crystals were grown in $4.0 \mathrm{M}$ sodium formate. Refinement of this condition using the Additive screen (Hampton Research, CA, USA) resulted in several conditions in which rod-like crystals could be grown (Table 1). All these different crystals belong to the same space group and were used at random to prepare soaks with several sugars. Prior to data collection, the crystals were transferred briefly into cryoprotectant composed of $4.0 \mathrm{M}$ sodium formate supplemented with $30 \%(v / v)$ glycerol and a saturated concentration of xylotriose (Megazyme, Bray, Ireland) [21]. Analogous soaking experiments were performed using xylotetraose (Megazyme), cellotetraose (Sigma-Aldrich, St. Louis, USA) and a mixture of arabinoxylo-oligosaccharides with an average degree of polymerization of 4 and an arabinose to xylose ratio of 0.5 (AXOS-4-0.5). These AXOS were derived from Wheat Pentosan Concentrate (WPC, Pfeifer \& Langen, Dormagen, Germany) and were kindly made available by Katrien Swennen (Laboratory of Food Chemistry and Biochemistry, K.U.Leuven, Belgium). In all cases, a saturated solution was used and soaking times ranged from 1 to 5 minutes.

Data from $B s \mathrm{AXH}-\mathrm{m} 2,3$ crystals soaked with xylotriose and xylotetraose were collected on beamline BW7a of DESY (EMBL-Hamburg, Germany) at cryogenic temperature and processed using the HKL suite of programs [22]. Data sets of native BsAXH-m2,3 crystals and those soaked with cellotetraose and AXOS-4-0.5, were collected on beamline X10SA of SLS (Villigen, Switzerland). These data were processed with iMosflm [23, 24] and scaled and merged 
with Scala [25]. For all structures, five percent of the observations were set aside for crossvalidation analysis. These sets are all equal to the set of observations of the data collected of the BsAXH-m2,3 crystal soaked with xylotriose.

\section{Structure solution and refinement}

All further computing was done using the CCP4 suite [26] unless stated otherwise. Due to the relatively low sequence similarity $[25 \%$ when comparing the catalytic domain with that of Cellvibrio japonicus $\alpha$-L-arabinanase (PDB-entry 1gyh) [15] and 35\% when comparing the CBM with Bacillus halodurans CBM family 6 (PDB-entry 1w9t) [27]] phasing the structure of $B S \mathrm{AXH}-\mathrm{m} 2,3$ by molecular replacement presented a challenging problem. Eventually, the structure of $B s \mathrm{AXH}-\mathrm{m} 2,3$ soaked with xylotriose was solved with an automatic approach: MrBUMP [28] implemented in the CCP4 interface, and using Phaser [29] as the underlying molecular replacement program, was used to position models of the two domains. An initial attempt at automated model re-building with ARP/wARP [30] using the sequence of $B s$ AXH$\mathrm{m} 2,3$ failed. Phase improvement of the molecular replacement solution was then carried out using artificial phase extension and dynamic density modification as implemented in the latest version of ACORN [31]. This step improved the phases sufficiently for ARP/wARP [30] to build and dock $93 \%$ of the residues. The coordinates were evaluated using Coot [32] and few corrections were made manually. The refined structure was then used to solve the structure of the native protein and the remaining substrate complexes using Phaser. After several cycles of refinement, residual positive density in the $F_{o}-F_{c}$ and $2 F_{o}-F_{c}$ electron density revealed the presence of several bound sugar molecules. These were inserted manually followed by further refinement and model building using Refmac5 [33] and Coot, respectively. The final structures 
were evaluated using Molprobity [34]. All data collection and refinement statistics are shown in Table 1. All figures were drawn using the program Pymol [35]. 


\section{Results and Discussion}

\section{The overall structure}

The structure of AXH-m2,3 from B. subtilis was determined by molecular replacement using the Cellvibrio japonicus $\alpha$-L-arabinanase (pdb-entry 1gyh) [15] and Bacillus halodurans CBM family 6 (pdb-entry 1w9t) [27] structures as search models for the catalytic domain and CBM, respectively. Several soaking experiments were performed to gain insight in the binding of substrate. All structures were determined to a maximum resolution ranging between 1.55 and $2.05 \AA$ and their refinement converged to good $R$-factors. This indicates that the structures can be used for a reliable analysis of the different interactions. Inspection of the electron density maps of the soaked structures revealed clear density for several sugar units. For the soaking experiments with xylotriose and xylotetraose, respectively three and four xylose units could be built in the electron density and the bound ligand atoms refined well with average thermal factors comparable to the values for all protein atoms. Also for the crystals soaked with cellotetraose and AXOS-4-0.5, there was clear density for four glucose and four xylose units, respectively, but no interpretable density for arabinose units could be observed for the structure soaked with AXOS4-0.5. For these structures, the thermal factors of the sugars are slightly higher compared to those for all protein atoms (Table 1).

Each asymmetric unit contains one $B S \mathrm{AXH}-\mathrm{m} 2,3$ monomer which is organized into two domains: the N-terminal catalytic domain which is a 5-bladed $\beta$-propeller fold common to all GH family 43 members, and a C-terminal $\beta$-sandwich domain, the CBM, analogous to CBM family 6 members (Figure 1). Superposition of the different structures revealed no large structural changes in the overall structure upon ligand binding. 
Unlike the $\beta$-xylosidase from G. stearothermophilus [14] and the bifunctional $\beta$-xylosidase/ $\alpha$-Larabinofuranosidase from $S$. ruminantium [17] BsAXH-m2,3 does not crystallize as a homotetramer, but as a single entity. This monomeric state of $B s \mathrm{AXH}-\mathrm{m} 2,3$ is in contrast to the quaternary structure of other two domain GH family 43 members for which the structure is known. The latter all occur as homotetramers which are composed of two dimers turned at $90^{\circ}$ against each other. The monomers forming the dimer are aligned antiparallel to one another such that the catalytic domain of one monomer interacts with the CBM of the neighboring domain. This arrangement results in a quaternary structure exhibiting 222 symmetry.

\section{The catalytic domain}

The catalytic domain forms a 5-bladed $\beta$-propeller, with the five $\beta$-sheets (marked I to V) radially arranged around a central water-filled tunnel like the blades of a propeller. Each $\beta$-sheet is built up of 4 antiparallel $\beta$-strands connected by hairpin turns, with the first strand being the innermost. All 5 innermost $\beta$-strands start at the same site, i.e. the entrance site, where the active site is located, and run almost parallel to one another. $\beta$-Strands 2 and 3 of the $5 \beta$-blades are connected by large loops lining the active site (Figure 1b). Together with the loop regions connecting $\beta$-strand 4 of one sheet to strand 1 of the next sheet, these loop regions probably have a role in determining the substrate specificity of $B s$ AXH-m2,3.

Unlike most propeller structures, $B s \mathrm{AXH}-\mathrm{m} 2,3$ does not show the classical molecular velcro which closes the propeller by incorporating the $\mathrm{N}$-terminus and $\mathrm{C}$-terminus in one blade of the propeller. However, some hydrogen bonds exist between the N-terminus and strand 4 of propeller blade $\mathrm{V}$. These bonds probably provide stabilization to the fold. This non-velcroed propeller has also been observed in the structure of other known GH family 43 members [15]. 


\section{The active site and substrate binding}

For members of GH family 43, three residues are essential for catalytic activity. For BsAXH$\mathrm{m} 2,3$, these residues could be identified by superposing the structure of $B s \mathrm{AXH}-\mathrm{m} 2,3$ with the structure of the $\beta$-xylosidase from G. stearothermophilus [14]. They are Asp 24, which is the general base; Glu 225, which is the general acid; and Asp 163, which is believed to play a role in the $\mathrm{p} K_{\mathrm{a}}$ modulation of the general acid and correct orientation of both the general acid and substrate. They are located on the innermost strands of $\beta$-blades I, IV and III, respectively, and point to the centre of the tunnel entrance site. Seen from the surface, they are situated in a small pocket - in which a single arabinose substituent would fit well - along an open cleft which allows binding of the xylan backbone. This arrangement of the active site is very suitable for the hydrolysis of arabinose substituents from monosubstituted xylose units and nicely illustrates why $B s$ AXH-m2,3 does not hydrolyze arabinose from disubstituted xylose units. The pocket is too small to allow binding of two arabinoses.

Since the crystals are grown from active BsAXH-m2,3, soaking with its substrate, arabinoxylooligosaccharides (AXOS) should result in hydrolysis of the arabinose substituents, and hence only the bound xylan backbone would probably be observed. Therefore, soaking experiments were not only performed with AXOS but also with short unsubstituted xylan chains to gain insight in the binding of the xylan backbone to BsAXH-m2,3. Three-dimensional complexes were obtained with xylotriose, xylotetraose, AXOS-4-0.5 and cellotetraose. The latter soaking experiment was performed to gain insight in the binding capacity of the CBM to cellulose since some CBM family 6 members are found to bind cellulose [19]. Surprisingly, cellotetraose was bound to the active site. To determine the direction of the sugar backbone, the difference electron 
density map was contoured at a high level to observe primarily the positions of the oxygen atoms. Interestingly, for the complex structures, a glycerol molecule (originating from the cryo solution) is located at the probable position of the target arabinose, and hence, gives us a hint about the interactions with the arabinose unit in this subsite. The different complex structures reveal several residues responsible for binding interactions with the xylan backbone. Since $B s \mathrm{AXH}-\mathrm{m} 2,3$ hydrolyzes substituents of the xylan backbone, the subsite numbering proposed by Davies and coworkers [36] can not be used to number the different binding subsites for the xylose units of the xylan backbone. So, for convenience, the here observed binding subsites are numbered I to IV starting from the xylose unit at the reducing end of the xylan backbone with the III subsite being equal to the -1 subsite in the numbering proposed by Davies and coworkers (Figure $2 a$ ). An arabinose substituent would be situated at the +1 subsite.

Superposition of the different complex structures shows no difference in the position of the bound sugars and glycerol molecules (Figure $2 \mathrm{~b}$ ). When superposing the unbound structure with the complex structure, the side chain of Asn 288 turns by $90^{\circ}$, away from the sugar. Apart from the xylose unit in the III subsite (from where the substituent is removed), only few hydrogen bonding interactions are observed between $B S \mathrm{AXH}-\mathrm{m} 2,3$ and the xylan backbone, of which all the xylose units are in the chair conformation (Figure 2). Two pronounced hydrophobic stacking interactions are observed with Phe 244 and Trp 160 and the xylose units in subsite II and IV, respectively, while the xylose unit in subsite I makes several hydrogen bonds with Gly 286. In the III subsite the $\mathrm{OH} 2$ of the xylose unit makes one hydrogen bond with Asn 288 (3.3 $\AA$ ) and two relatively strong hydrogen bonds with the general acid, Glu 225 (2.6 and $3.0 \AA$ ), while the OH3 makes one hydrogen bond with Glu 225 (3.6 A). The extra carbonyl group of the glucose units from cellotetraose does not seem to make any additional interaction with $B s \mathrm{AXH}-\mathrm{m} 2,3$ in 
comparison with xylotetraose. The binding of cellotetraose to the active site is probably an artifact based on the structural relationship between the xylopyranose and glucopyranse ring since this binding has no physiological meaning, i.e., arabinocellulose does not occur in nature. In the II and IV subsite, binding is solely based on hydrophobic stacking interactions. Since, in contrast to an extensive hydrogen bonding network, hydrophobic stacking interactions allow more positional freedom, these interactions may account for the observation that either substituents at the $O-2$ or $O-3$ hydroxyl position of the xylose can be hydrolyzed. This property of $B s \mathrm{AXH}-\mathrm{m} 2,3$ needs some positional flexibility to allow correct positioning of the glycosidic bond with regard to the catalytic triad. Further studies involving soaking experiments with inactive $B s \mathrm{AXH}-\mathrm{m} 2,3$ and specific AXOS only substituted at their $O-2$ or $O-3$ hydroxyl position are required to confirm the above hypothesis.

Since the hydroxyl groups from glycerol usually take similar positions to the hydroxyl groups from sugars, the interactions between the glycerol in the catalytic pocket and BsAXH-m2,3 give us an idea of the interactions with the arabinose unit in the catalytic pocket. Hydrogen bonds are formed with the three catalytic residues (Glu 225, Asp 163 and Asp 24) and Arg 321 (Table 2). In analogy with other GH family 43 members, Trp 101, which is part of the invariant Trp-AlaPro element, probably makes a hydrophobic stacking interaction with the arabinose unit [14].

\section{The carbohydrate binding module}

In analogy with other CBM family 6 members, the CBM of $B s \mathrm{AXH}-\mathrm{m} 2,3$ forms a $\beta$-sandwich fold consisting of five antiparallel $\beta$-strands on one face and four antiparallel $\beta$-strands on the other face (Figure 3). During refinement, the electron density maps revealed residual density for metal ions at two positions (Figure 3 ). The first metal ion could be modeled as a calcium ion and 
is hepta-coordinated by the side chains of Glu 359, Glu 361, Asn 383 and Asp 480. The coordination is completed by the backbone carbonyl oxygens of Gln 384 and Asp 480. The position and nature of this ion is identical to those found in other known CBM family 6 members and probably makes it a structural ion $[14,19,27,37,38]$. It is likely to be already incorporated during protein folding since no calcium ions were used during the purification and crystallization of $B s \mathrm{AXH}-\mathrm{m} 2,3$, supporting the idea of it being a structural ion. The coordination of the second ion - modeled as a sodium - involves the side chains of Gln 390 and Asp 393. This coordination is completed by the main chain carbonyl oxygens of Arg 368 and Ser 388 and two water molecules. This metal ion corresponds to the sodium ion observed in the CBM family 6 of Bacillus halodurans laminarinase and to the sodium ion observed in the CBM family 6 of Cellvibrio mixtus endoglucanase 5A [27, 38]. In contrast to the calcium ion, sodium might have been incorporated during the crystallization of BsAXH-m2,3 since the precipitant and cryo solutions contain sodium ions.

Soaking experiments showed that neither cellotetraose nor xylo-oligosaccharides were bound to the CBM. This can tentatively be explained as follows. CBMs of family 6 contain two clefts which play a role in sugar binding (Figure 4a). Cleft A is found in the loop region connecting the inner and outer $\beta$-sheets of the $\beta$-sandwich and resembles the sugar binding sites of lectins [37], while cleft $\mathrm{B}$ is located on the concave surface of one $\beta$-sheet and has a similar location as the binding sites of CBMs from several other families [38]. When comparing cleft A of BsAXH$\mathrm{m} 2,3$ with several other CBMs of family $6, B s \mathrm{AXH}-\mathrm{m} 2,3$ is clearly lacking the two aromatic residues which play a key role in ligand binding [39]. The amino acids at the equivalent position are Ser 388 and Ala 445 (Figure 4b). Cleft B is lined with two aromatic residues, Trp 394 and Phe 465, which is in agreement with other CBMs belonging to family 6. However, in analogy 
with the CBM family 6 from Clostridium thermocellum xylanase 11A [19], the CBM family 6 from Bacillus halodurans laminarinase [27] and the CBM family 6 from Clostridium stercorarium xylanase [37], a loop region covers this cleft making it inaccessible for sugar (Figure 4c). These findings may explain the absence of binding of xylo-oligosaccharides and cellotetraose to the CBM of BsAXH-m2,3, since both binding clefts are structurally incapable of binding sugar chains. This CBM probably lost its carbohydrate binding function during evolution. The loss of carbohydrate binding capacity is also seen for CBMs of family 48. This family of CBMs is related to the starch-binding domains belonging to CBM families 20 and 21. Members of the latter families have binding sites which are lined with aromatic residues [40]. In analogy to cleft $\mathrm{A}$ of the CBM of $B s \mathrm{AXH}-\mathrm{m} 2,3$ the carbohydrate binding capacity of the CBM48 of glycosyltrehalose trehalohydrolase from Sulfolobus solfatoricus is also lost due to mutations of the aromatic residues which are responsible for carbohydrate binding [41].

\section{Comparison of BsAXH-m2,3 with other GH family 43 structures}

The availability of the structure of this AXH-m2,3 and of other GH family 43 enzymes, in complex with their substrates provides insight in how these structurally related enzymes are fine tuned towards different substrate specificities. Indeed, despite their similar structures, reaction mechanisms and catalytic residue positioning [10], $\alpha$-L-arabinanases and $\beta$-xylosidases display exo-activity, whereas $\mathrm{AXH}-\mathrm{m} 2,3$ is a substituent releasing enzyme.

Superposition of substrate bound structures of $\alpha$-L-arabinanase from Cellvibrio japonicus (pdbentry 1gye) [15], $\beta$-xylosidase from Geobacillus stearothermophilus (pdb-entry 2exk) [14] and $B S \mathrm{AXH}-\mathrm{m} 2,3$ in complex with xylotetraose reveals a different orientation of the sugar backbone. The xylan backbone bound to $B s \mathrm{AXH}-\mathrm{m} 2,3$ is perpendicular to the arabinose and xylose 
backbones bound to $\alpha$-L-arabinanase and $\beta$-xylosidase respectively (Figure 5a). A closer look at the binding pocket of the three enzymes reveals a number of differences between them (Figure 5b). For $\alpha$-L-arabinanase and $\beta$-xylosidase, the loop region connecting $\beta$-strands 2 and 3 of $\beta$ sheet II are much longer in comparison to the same loop region of $B s \mathrm{AXH}-\mathrm{m} 2,3$. In addition, their loop region connecting $\beta$-strands 2 and 3 of $\beta$-sheet IV is flipped in comparison to BsAXH$\mathrm{m} 2,3$. Thus, for $\alpha$-L-arabinanase and $\beta$-xylosidase, the loop regions connecting $\beta$-strands 2 and 3 of both $\beta$-sheets II and IV prevent the enzyme from hydrolyzing substituents from the backbone. Superposition of $B s \mathrm{AXH}-\mathrm{m} 2,3$ and $\beta$-xylosidase also reveals a different position of their CBMs with respect to the catalytic domain (Figure $5 \mathrm{c}$ ). In $\beta$-xylosidase the CBM plays an important role in restricting the possible length of the incoming substrate by closing off a portion of the active site to form a pocket $[14,17]$. This results in an exo mode of action and the release of only one xylose unit, while for $\alpha$-L-arabinanase, which has no CBM, arabinotriose units are released. For $B s \mathrm{AXH}-\mathrm{m} 2,3$, due to its position, the CBM does not seem to have an influence on the activity of the enzyme; there are no residues of its CBM which are involved in the active site or in the entrance to the active site. Therefore, removing the CBM would probably not have an influence on the specificity of BsAXH-m2,3.

In conclusion, soaking experiments of $B S A X H-m 2,3$ crystals with several sugars not only explained the specific activity of $B s \mathrm{AXH}-\mathrm{m} 2,3$, but also showed some remarkable differences in substrate binding between GH family 43 enzymes. Since substrate binding is mostly based on hydrophobic stacking interactions, this probably gives the necessary positional freedom to hydrolyze arabinose substituents either at the $O-2$ or $O-3$ hydroxyl position of the xylose. The observation of both exo-activity and the release of substituents within GH family 43 enzymes, 
results from differences at the binding pocket which account for a different binding orientation of the sugar backbone.

\section{Acknowledgements}

17

Licenced copy. Copying is not permitted, except with prior permission and as allowed by law. (c) 2008 The Authors Journal compilation (c) 2008 Biochemical Society 
We like to thank the staff of the EMBL/DESY Hamburg Outstation and the SLS for the provision of synchrotron facilities, skillful technical assistance and financial support through the I3 contract with the European Commission for support of access for external users of the EMBL/DESY Hamburg Outstation. This work was funded by the Flemish IWT (Instituut voor de aanmoediging van Innovatie door Wetenschap en Technologie in Vlaanderen, IMPAXOS SBO project funding).

\section{References}


1 Gray, K. A., Zhao, L. and Emptage, M. (2006) Bioethanol. Curr. Opin. Chem. Biol. 10, 141-146

2 Saha, B. C. (2003) Hemicellulose bioconversion. J. Ind. Microbiol. Biotechnol. 30, 279291

3 Jeffries, T. W. (1994) Biodegradation of lignin and hemicelluloses. In Biochemistry of Microbial Degradation. (Ratledge, C., ed.). pp. 233-277, Kluwer Academic Publishers, The Netherlands

4 Pitson, S. M., Voragen, A. G. J. and Beldman, G. (1996) Stereochemical course of hydrolysis catalyzed by arabinofuranosyl hydrolases. FEBS Lett. 398, 7-11

5 Kormelink, F. J. M., Leeuwen, M. J. F. S.-v., Wood, T. M. and Voragen, A. G. J. (1991) $(1,4)-$ beta-D-arabinoxylan arabinofuranohydrolase - a novel enzyme in the bioconversion of arabinoxylan. Appl. Microbiol. Biotechnol. 35, 231-232

6 Laere, K. M. J. V., Beldman, G. and Voragen, A. G. J. (1997) A new arabinofuranohydrolase from Bifidobacterium adolescentis able to remove arabinosyl residues from double-substituted xylose units in arabinoxylan. Appl. Microbiol. Biotechnol. 47, 231-235

7 Laere, K. M. J. V., Voragen, C. H. L., Kroef, T., Broek, L. A. M. V. d., Beldman, G. and Voragen, A. G. J. (1999) Purification and mode of action of two different arabinoxylan arabinofuranohydrolases from Bifidobacterium adolescentis DSM 20083. Appl. Microbiol. Biotechnol. 51, 606-613

8 Ferré, H., Broberg, A., Duus, J. O. and Thomsen, K. K. (2000) A novel type of arabinoxylan arabinofuranohydrolase isolated from germinated barley - Analysis of substrate preference and specificity by nano-probe NMR. Eur. J. Biochem. 267, 6633-6641 

database approach. In Recent Advances in Carbohydrate Bioengineering (Gilbert, H. J., Davies, G., Henrissat, B. and Svensson, B., eds.). pp. 3-12, The Royal Society of Chemistry, Cambridge 10 Davies, G. and Henrissat, B. (1995) Structures and mechanisms of glycosyl hydrolases. Structure. 3, 853-859

11 Hövel, K., Shallom, D., Niefind, K., Belakhov, V., Shoham, G., Baasov, T., Shoham, Y. and Schomburg, D. (2003) Crystal structure and snapshots along the reaction pathway of a family 51 alpha-L-arabinofuranosidase. EMBO J. 22, 4922-4932

12 Miyanaga, A., Koseki, T., Matsuzawa, H., Wakagi, T., Shoun, H. and Fushinobu, S. (2004) Crystal structure of a family 54 alpha-L-arabinofuranosidase reveals a novel carbohydrate-binding module that can bind arabinose. J. Biol. Chem. 279, 44907-44914

13 Bourgois, T. M., Van Craeyveld, V., Van Campenhout, S., Courtin, C. M., Delcour, J. A., Robben, J. and Volckaert, G. (2007) Recombinant expression and characterization of XynD from Bacillus subtilis subsp. subtilis ATCC 6051: a GH 43 arabinoxylan arabinofuranohydrolase. Appl. Microbiol. Biotechnol. 75, 1309-1317

14 Brüx, C., Ben-David, A., Shallom-Shezifi, D., Leon, M., Karsten, N., Gil, S., Yuval, S. and Dietmar, S. (2006) The structure of an inverting GH43 beta-xylosidase from Geobacillus stearothermophilus with its substrate reveals the role of the three catalytic residues. J. Mol. Biol. 359, 97-109

15 Nurizzo, D., Turkenburg, J. P., Charnock, S. J., Roberts, S. M., Dodson, E. J., McKie, V. A., Taylor, E. J., Gilbert, H. J. and Davies, G. J. (2002) Cellvibrio japonicus alpha-L-arabinase 43A has a novel five-blade beta-propeller fold. Nat. Struct. Biol. 9, 665-668 
16 Yamaguchi, A., Tada, T., Wada, K., Nakaniwa, T., Kitatani, T., Sogabe, Y., Takao, M., Sakai, T. and Nishimura, K. (2005) Structural basis for thermostability of endo-1,5-alpha-Larabinanase from Bacillus thermodenitrificans TS-3. J. Biochem. 137, 587-592

17 Brunzelle, J. S., Jordan, D. B., McCaslin, D. R., Olczak, A. and Wawrzak, Z. (2008) Structure of the two-subsite beta-D-xylosidase from Selenomonas ruminantium in complex with 1,3-bis[tris(hydroxymethyl)methylamino]propane. Arch. Biochem. Biophys. 474, 157-166

18 Beisel, H.-G., Kawabata, S.-i., Iwanaga, S., Huber, R. and Bode, W. (1999) Tachylectin2: crystal structure of a specific GlcNAc/GalNAc-binding lectin involved in the innate immunity host defense of the Japanese horseshoe crab Tachypleus tridentatus. EMBO J. 18, 2313-2322

19 Czjzek, M., Bolam, D. N., Mosbah, A., Allouch, J., Fontes, C. M. G. A., Ferreira, L. M. A., Bornet, O., Zamboni, V., Darbon, H., Smith, N. L., Black, G. W., Henrissat, B. and Gilbert, H. J. (2001) The location of the ligand-binding site of carbohydrate-binding modules that have evolved from a common sequence is not conserved. J. Biol. Chem. 276, 48580-48587

20 Boraston, A. B., Bolam, D. N., Gilbert, H. J. and Davies, G. J. (2004) Carbohydratebinding modules: fine-tuning polysaccharide recognition. Biochem. J. 382, 769-781

21 Vandermarliere, E., Bourgois, T. M., Van Campenhout, S., Strelkov, S. V., Volckaert, G., Delcour, J. A., Courtin, C. M. and Rabijns, A. (2007) Crystallization and preliminary X-ray analysis of an arabinoxylan arabinofuranohydrolase from Bacillus subtilis. Acta Cryst. F63, 692694

22 Otwinowski, Z. and Minor, W. (1997) Processing of X-ray diffraction data collected in oscillation mode. In Methods in enzymology, volume 276: Macromolecular crystallography, part A (Carter, C. W. and Sweet, J. R. M., eds.). pp. 307-326, Academic Press, New York 
23 Leslie, A. (2006) The integration of macromolecular diffraction data. Acta Crystallogr. D62, 48-57

24 Powell, H., Leslie, A. and Battye, G. (2007) Mosflm 7.0.1 and its new interface iMosflm 0.5.3. In CCP4 Newsletter ed.)^eds.)

25 Evans, P. (2006) Scaling and assessment of data quality. Acta Crystallogr. D62, 72-82

26 Collaborative Computational Project Number 4. (1994) The CCP4 suite: programs for protein crystallography. Acta Crystallogr. D50, 760-763

27 Lammerts van Bueren, A., Morland, C., Gilbert, H. J. and Boraston, A. B. (2005) Family 6 carbohydrate binding modules recognize the non-reducing end of beta-1,3-linked glucans by presenting a unique ligand binding surface. J. Biol. Chem. 280, 530-537

28 Keegan, R. M. and Winn, M. D. (2007) Automated search-model discovery and preparation for structure solution by molecular replacement. Acta Crystallogr. D63, 447-457

29 McCoy, A. J., Grosse-Kunstleve, R. W., Adams, P. D., Winn, M. D., Storoni, L. C. and Read, R. J. (2007) Phaser crystallographic software. J. Appl. Cryst. 40, 658-674

30 Perrakis, A., Morris, R. and Lamzin, V. S. (1999) Automated protein model building combined with iterative structure refinement. Nat. Struct. Biol. 6, 458-463

31 Jia-Xing, Y., Woolfson, M. M., Wilson, K. S. and Dodson, E. J. (2005) A modified ACORN to solve protein structures at resolutions of 1.7 Armstrong or better. Acta Crystallogr.

D61, 1465-1475

32 Emsley, P. and Cowtan, K. (2004) Coot: model-building tools for molecular graphics. Acta Crystallogr. D60, 2126-2132

33 Murshudov, G. N., Vagin, A. A. and Dodson, E. J. (1997) Refinement of molecular structures by the maximum-likelihood method. Acta Crystallogr. D53, 240-255 
34 Lovell, S. C., Davis, I. W., Arendall, B., de Bakker, P. I. W., Word, J. M., Prisant, M. G., Richardson, J. S. and Richardson, D. C. (2003) Structure validation by C-alpha geometry: phi, psi, and C-beta deviation. Proteins. 50, 437-450

35 Delano, W. L. (2004) The PyMol molecular graphics system. DeLano Scientific, LLC, Sanc Carlos, CA

36 Davies, G. J., Wilson, K. S. and Henrissat, B. (1997) Nomenclature for sugar-binding subsites in glycosyl hydrolases. Biochem. J. 321, 557-559

37 Boraston, A. B., Notenboom, V., Warren, R. A. J., Kilburn, D. G., Rose, D. R. and Davies, G. J. (2003) Structure and ligand binding of carbohydrate-binding module CsCBM6-3 reveals similarities with fucose-specific lectins and "galactose-binding" domains. J. Mol. Biol. 327, 659-669

38 Pires, V. M. R., Henshaw, J. L., Prates, J. A. M., Bolam, D. N., Ferreira, L. M. A., Fontes, C. M. G. A., Henrissat, B., Planas, A., Gilbert, H. J. and Czjzek, M. (2004) The crystal structure of the family 6 carbohydrate binding module from Cellvibrio mixtus endoglucanase 5A in complex with oligosaccharides reveals two distinct binding sites with different ligand specificities. J. Biol. Chem. 279, 21560-21568

39 Henshaw, J. L., Bolam, D. N., Pires, V. M. R., Czjzek, M., Henrissat, B., Ferreira, L. M. A., Fontes, C. M. G. A. and Gilbert, H. J. (2004) The family 6 carbohydrate binding module CmCBM6-2 contains two ligand-binding sites with distinct specificities. J. Biol. Chem. 279, 21552-21559

40 Machovic, M. and Janecek, S. (2006) Starch-binding domains in the post-genomic era. Cell. Mol. Life Sci. 63, 2710-2724 
41 Feese, M. D., Kato, Y., Tamada, T., Kato, M., Komeda, T., Miura, Y., Hirose, M., Hondo, K., Kobayashi, K. and Kuroki, R. (2000) Crystal structure of glycosyltrehalose trehalohydrolase from the hyperthermophilic Archaeum Sulfolobus solfataricus. J. Mol. Biol.

301, 451-464 
Table 1: Data collection and refinement statistics.

\begin{tabular}{|c|c|c|c|c|c|c|}
\hline & $\mathrm{AXH}$ & $\begin{array}{c}\text { AXH } \\
\text { xylotriose }\end{array}$ & $\begin{array}{c}\text { AXH } \\
\text { xylotetraose }\end{array}$ & $\begin{array}{c}\text { AXH } \\
\text { AXOS-4-0.5 }\end{array}$ & $\begin{array}{c}\text { AXH } \\
\text { cellotetraose }\end{array}$ & \\
\hline Additive & $\begin{array}{c}30 \%(\mathrm{w} / \mathrm{v}) \\
\text { sucrose }\end{array}$ & $\begin{array}{l}\text { 1.0 M Lithium } \\
\text { Chloride }\end{array}$ & & $\begin{array}{l}0.1 \mathrm{M} \text { betaine } \\
\text { hydrochloride }\end{array}$ & $\begin{array}{c}30 \%(\mathrm{w} / \mathrm{v}) \\
\text { sucrose }\end{array}$ & \\
\hline \multicolumn{7}{|l|}{ Data collection } \\
\hline Wavelength (A) & 0.9785 & 1.0788 & 1.0322 & 0.9785 & 1.0011 & \\
\hline Source & SLS X10SA & DESY BW7a & DESY BW7a & SLS X10SA & SLS X10SA & \\
\hline Resolution range $(\AA)$ & $\begin{array}{c}30-2.0(2.11- \\
2.0)\end{array}$ & $\begin{array}{c}50-1.55(1.58- \\
1.55)\end{array}$ & $\begin{array}{c}50-2.05(2.09- \\
2.05)\end{array}$ & $30-1.8(1.9-1.8)$ & $30-1.8(1.9-1.8)$ & \\
\hline \multicolumn{7}{|l|}{ Reflections } \\
\hline Observed & 156621 & 258631 & 150581 & 390182 & 306957 & $\bar{\infty}_{0}^{-\infty}$ \\
\hline Unique & 35773 (4966) & $76939(3868)$ & $33537(1596)$ & $50933(7349)$ & $45414(6055)$ & ○ \\
\hline Completeness (\%) & $96.4(93.2)$ & $98.1(99.9)$ & $97.0(93.9)$ & $99.9(99.8)$ & $92.0(85.4)$ & m \\
\hline Mean $I / \sigma(I)$ & $14.1(3.9)$ & $14.0(3.9)$ & $22.4(8.0)$ & $19.9(6.1)$ & $19.0(5.4)$ & $\stackrel{\mathrm{J}}{\mathrm{y}}$ \\
\hline Multiplicity & $4.4(4.2)$ & $3.4(3.1)$ & $4.5(4.2)$ & $7.7(7.5)$ & $6.8(6.6)$ & 으 \\
\hline$R_{\mathrm{sym}}(\%)^{a}$ & $10.2(33.3)$ & $8.3(32.2)$ & $4.6(13.7)$ & $8.1(27.9)$ & $8.6(29.2)$ & 은 \\
\hline \multicolumn{7}{|l|}{ Crystal } \\
\hline Space group & $P 2{ }_{1} 2_{1} 2_{1}$ & $P 2_{1} 2_{1} 2_{1}$ & $P 2{ }_{1} 2_{1} 2_{1}$ & $P 2_{1} 2_{1} 2_{1}$ & $P 2{ }_{1}{ }_{1} 2_{1}$ & 0 \\
\hline Unit-cell parameters & $a=67.6 \AA$ & $a=68.7 \AA$ & $a=67.5 \AA$ & $a=67.8 \AA$ & $a=68.0 \AA$ & \\
\hline & $b=74.4 \AA$ & $b=73.7 \AA$ & $b=72.4 \AA$ & $b=74.2 \AA$ & $b=73.1 \AA$ & \\
\hline & $c=107.0 \AA$ & $c=106.5 \AA$ & $c=106.9 \AA$ & $c=107.3 \AA$ & $c=105.7 \AA$ & 品 \\
\hline \multicolumn{7}{|l|}{ Refinement } \\
\hline$R_{\text {work }}(\%)^{b}$ & 16.8 & 16.4 & 15.1 & 15.5 & 15.1 & $\ddot{\varpi}$ \\
\hline$R_{\text {free }}(\%)^{c}$ & 21.3 & 19.0 & 17.4 & 19.0 & 17.5 & 岁 \\
\hline \multicolumn{7}{|l|}{$\mathrm{Rms}^{d}$ deviations } \\
\hline Bond lengths $(\AA)$ & 0.016 & 0.010 & 0.012 & 0.015 & 0.012 & $\underline{0}$ \\
\hline Bond angles $\left({ }^{\circ}\right)$ & 1.50 & 1.22 & 1.50 & 1.44 & 1.50 & $\overline{\mathscr{N}}$ \\
\hline \multicolumn{7}{|l|}{ Number of atoms } \\
\hline Protein & 3671 & 3674 & 3675 & 3682 & 3661 & 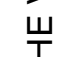 \\
\hline Solvent & 311 & 579 & 446 & 407 & 440 & $\stackrel{I}{\vdash}$ \\
\hline Ligand & & 28 & 37 & 28 & 45 & 5 \\
\hline \multicolumn{7}{|l|}{ Average $B$-factor $\left(\AA^{2}\right)$} \\
\hline Main chain & 16.1 & 13.9 & 12.5 & 14.1 & 14.9 & $\underline{\omega}$ \\
\hline Side chain & 16.0 & 14.5 & 12.5 & 14.1 & 15.4 & $\stackrel{\omega}{T}$ \\
\hline Solvent & & 29.1 & 24.0 & 24.1 & 32.0 & $\stackrel{\perp}{\vdash}$ \\
\hline Ligand & & 16.7 & 18.1 & 52.3 & 60.0 & \\
\hline PDB-entry & $3 \mathrm{C} 7 \mathrm{E}$ & $3 \mathrm{C} 7 \mathrm{~F}$ & $3 \mathrm{C} 7 \mathrm{G}$ & $3 \mathrm{C} 7 \mathrm{H}$ & $3 \mathrm{C} 7 \mathrm{O}$ & \\
\hline
\end{tabular}

Values in parentheses are for the highest resolution shell.

${ }^{a} R_{\text {sym }}=\sum|I-(I)| / \sum I$.

${ }^{b} R=\sum|| F_{o}|-| c\left|/ \sum\right| F_{c} \mid$

${ }^{c}$ The $R_{\text {free }}$ was calculated with $5 \%$ of the data excluded from structure refinement.

${ }^{d}$ Root mean square. 
Table 2: Interactions between $B s A X H-m 2,3$ and xylotetraose.

\begin{tabular}{lccc}
\hline Subsite & Substrate atom & Protein atom & Distance $(\AA)$ \\
\hline I subsite & O2 & Gly 286 N & 3.2 \\
& O2 & Gly 286 CO & 3.5 \\
& O3 & Gly 286 CO & 3.6 \\
II subsite & & Phe 244 & Hydrophobic stacking \\
III subsite (-1 subsite) & O2 & Asn 288 N $\delta 2$ & 3.3 \\
& O2 & Glu 225 O 22 & 2.6 \\
& O2 & Glu 225 O $\varepsilon$ & 3.0 \\
IV subsite & O3 & Glu 225 O $\varepsilon 1$ & 3.6 \\
+1 subsite (glycerol) & & & \\
& & Trp 160 & Hydrophobic stacking \\
& O1 & Glu 225 O $\varepsilon 1$ & 3.4 \\
& O1 & Asp 163 O $\delta 2$ & 3.3 \\
& O1 & Asp 163 O $\delta 1$ & 2.5 \\
& O3 & Asp 24 O $\delta 1$ & 3.5 \\
& O3 & Asp 24 O $\delta 2$ & 2.7 \\
& O3 & Arg 321 NH2 & 2.9 \\
& O3 & Arg 321 NH1 & 2.8 \\
& & Trp 101 & Hydrophobic stacking \\
\hline
\end{tabular}




\section{Figure legends}

\section{Figure 1}

Overall structure of $B s \mathrm{AXH}-\mathrm{m} 2,3$. The catalytic domain is shown in blue, while the CBM is shown in green. The three catalytic residues are shown in ball and stick representation. a) Top view showing the numbering of the $5 \beta$-blades (I to V). b) Side view showing the position of the active site surrounded by the long loops connecting $\beta$-strand 2 and 3 of each $\beta$-blade.

\section{Figure 2}

Detailed view of the active site of $B s \mathrm{AXH}-\mathrm{m} 2,3$. a) The interactions between xylotetraose (white) and AXH-m2,3 are shown with hydrogen bonds represented as red dotted lines. The glycerol molecule occupying the catalytic pocket is shown in grey. The catalytic residues are in orange. b) Superposition of the substrate molecules in the active site. The structure soaked with xylotriose is shown in blue, the one soaked with xylotetraose in pink, with AXOS-4-0.5 in green and the structure soaked with cellotetraose is shown in grey.

\section{Figure 3}

Overall structure of the CBM and its bound metal ions. a) Overall view of the CBM. The sodium ion is shown in purple, the calcium ion in orange. b) Detailed view of the interactions between $B s \mathrm{AXH}-\mathrm{m} 2,3$ and the calcium ion. c) Detailed view of the interactions between $B s \mathrm{AXH}-\mathrm{m} 2,3$ and the sodium ion. The waters involved in coordinating the sodium ion are shown as red balls. 


\section{Figure 4}

Structural differences at the binding clefts of CBM family 6 members. a) Cartoon representation of the CBM of $B s \mathrm{AXH}-\mathrm{m} 2,3$ showing the position of both binding cleft A and B. b) Detail of binding cleft A. The two aromatic residues with a key role in sugar binding are shown in ball and stick representation. The CBM6 of BsAXH-m2,3 is shown in green, the CBM6 of Clostridium thermocellum xylanase 11A (pdb-entry 1gmm) [19] is shown in purple, the CBM6 of Cellvibrio mixtus endoglucanase 5A (pdb-entry 1uyz) [38] is shown in blue, the CBM6 of Bacillus haludorans laminarinase (pdb-entry 1w9t) [27] is shown in orange and the CBM6 of Clostridium stercorarium xylanase (pdb-entry 1nae) [37] is shown in grey. c) Detail of binding cleft B. The two aromatic residues which are important for sugar binding are shown in ball and stick representation. The CBM6 of BsAXH-m2,3 is shown in green and the CBM6 of Clostridium thermocellum xylanase 11A (pdb-entry $1 \mathrm{gmm}$ ) [19] is shown in purple.

\section{Figure 5}

Structural differences at the active site of several GH family 43 members. a) Detail of the superposition of the active site of $B s \mathrm{AXH}-\mathrm{m} 2,3$ (blue), $\alpha$-L-arabinanase from Cellvibrio japonicus (purple) and $\beta$-xylosidase from Geobacillus stearothermophilus (green) in complex with their substrate, showing the perpendicular orientation of the substrate. b) Cartoon representation of the superposition of $B S \mathrm{AXH}-\mathrm{m} 2,3$ (blue) in complex with xylotetraose (ball and stick), $\alpha$-L-arabinanase (purple) and $\beta$-xylosidase (green). The loop regions of $\alpha$-Larabinanase and $\beta$-xylosidase clashing with the xylotetraose are pointed. c) Superposition of $B S \mathrm{AXH}-\mathrm{m} 2,3$ and $\beta$-xylosidase demonstrating the different position of their CBM relative to the catalytic domain. 


\section{Figure 1}

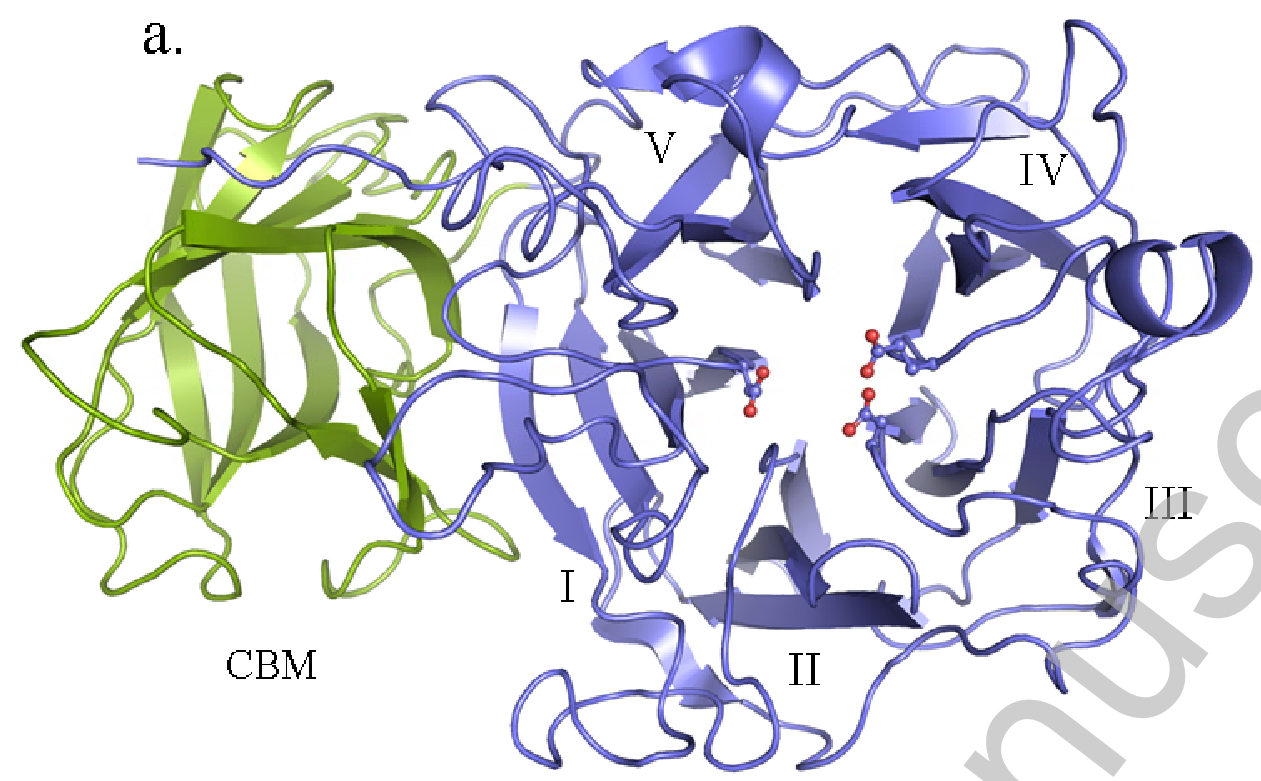

Catalytic domain

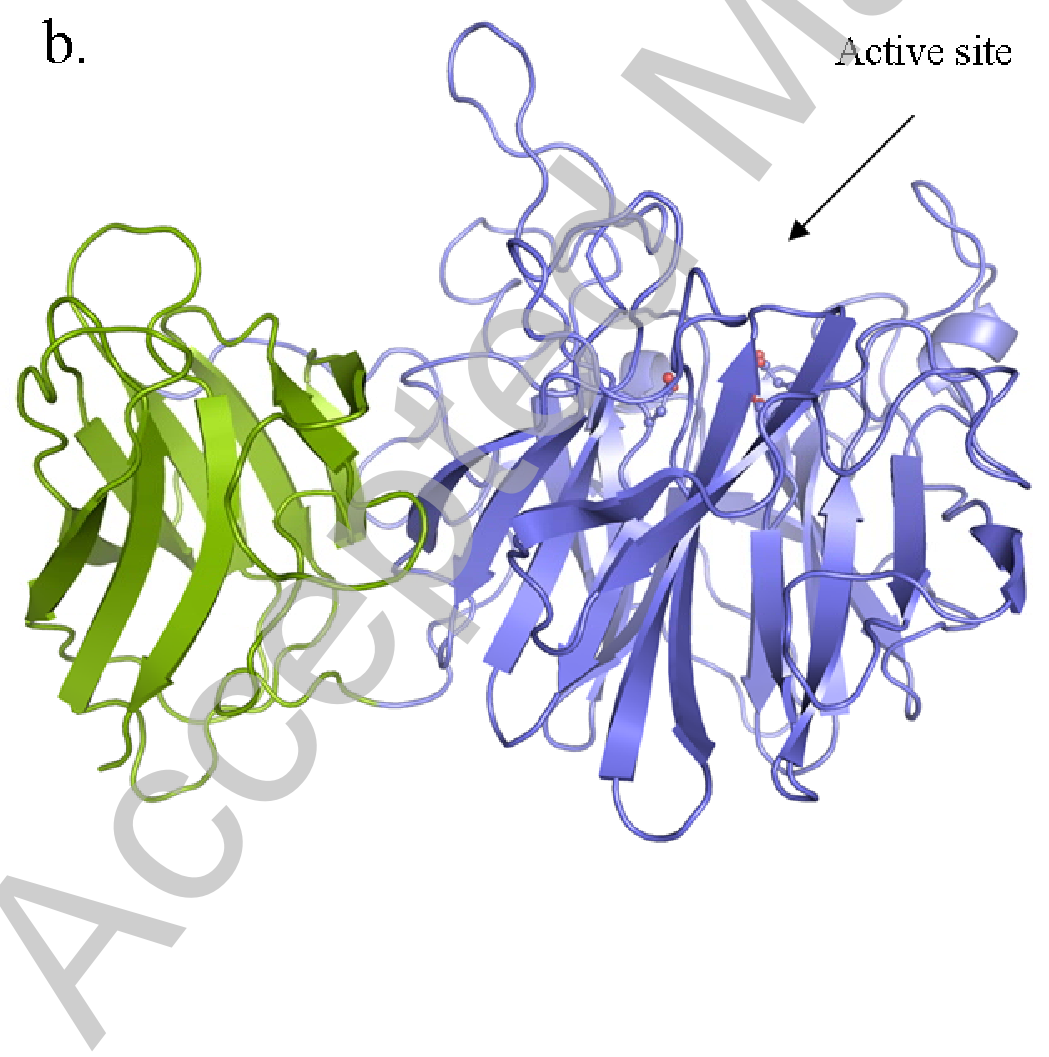




\section{Figure 2}
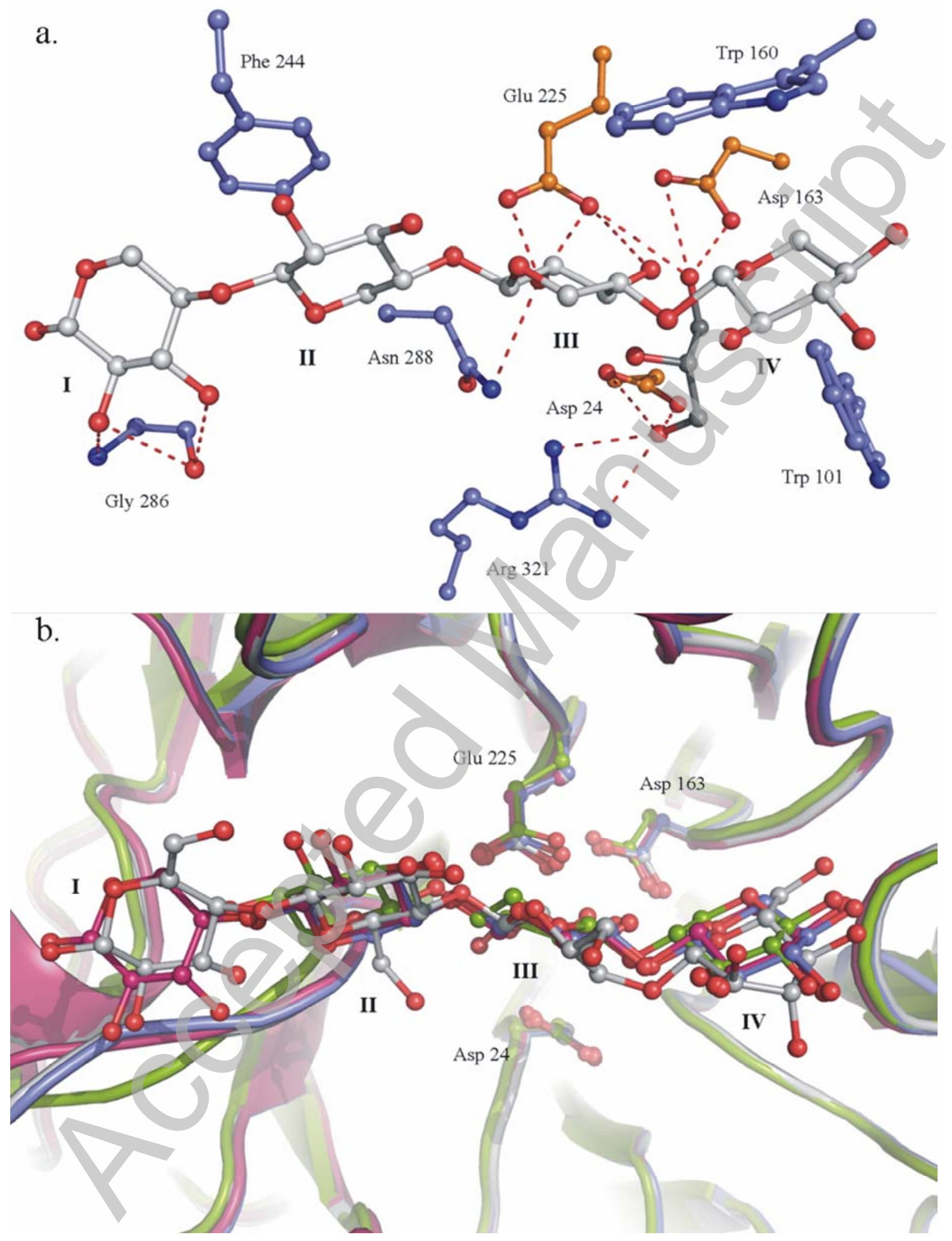


\section{Figure 3}
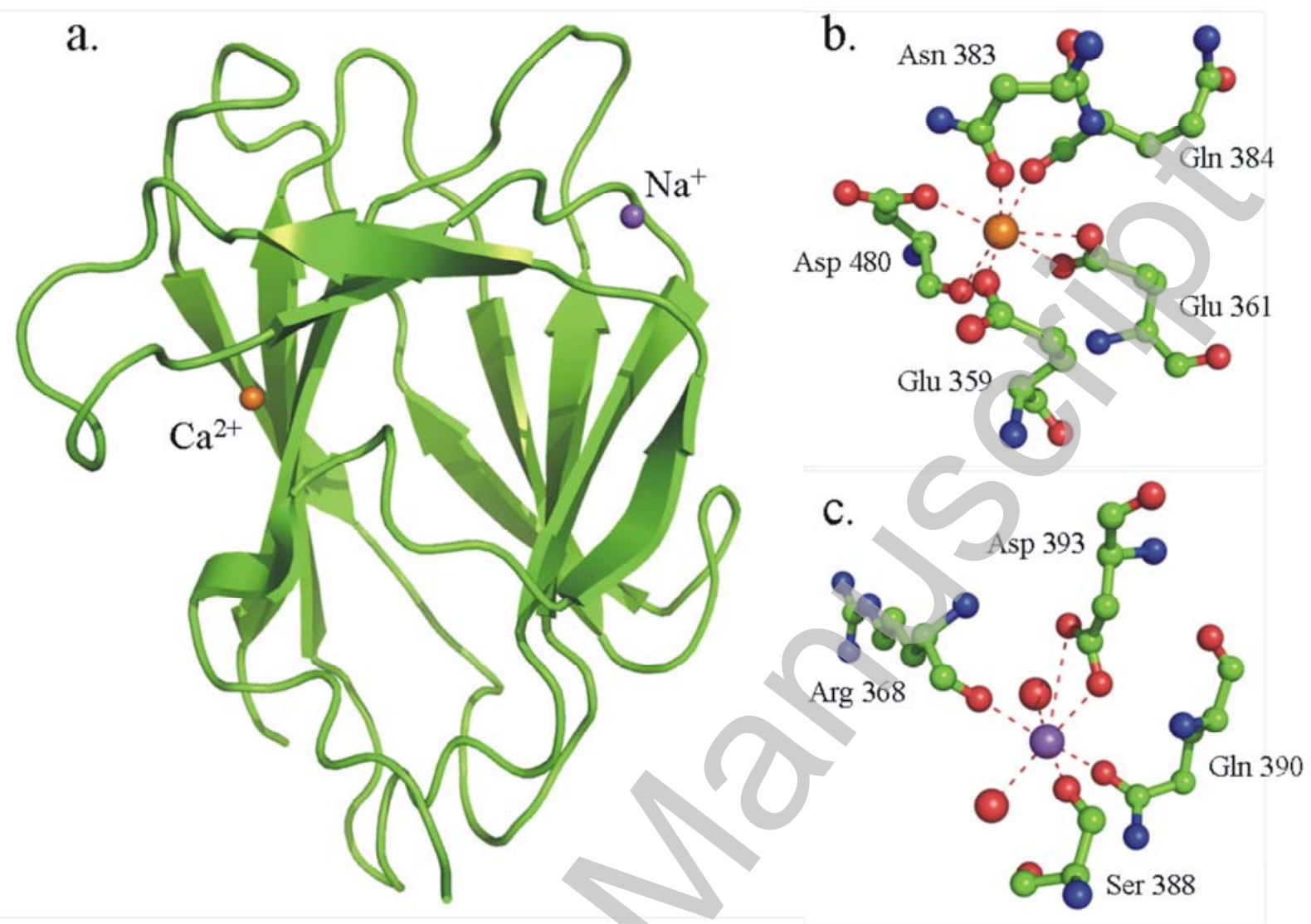


\section{Figure 4}

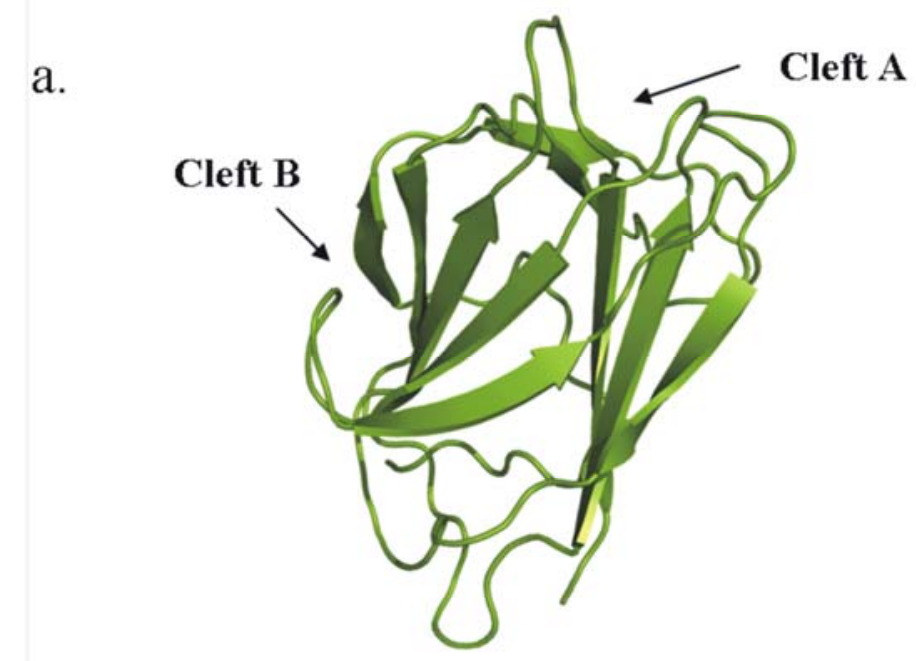

b.

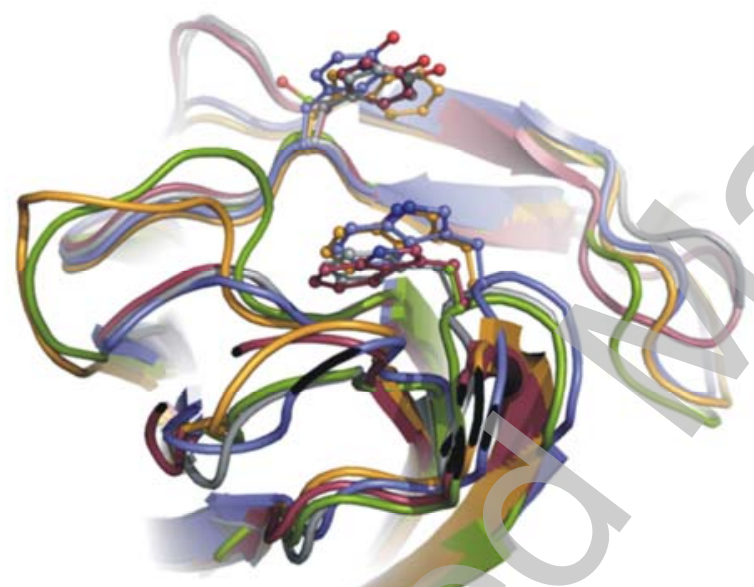




\section{Figure 5}

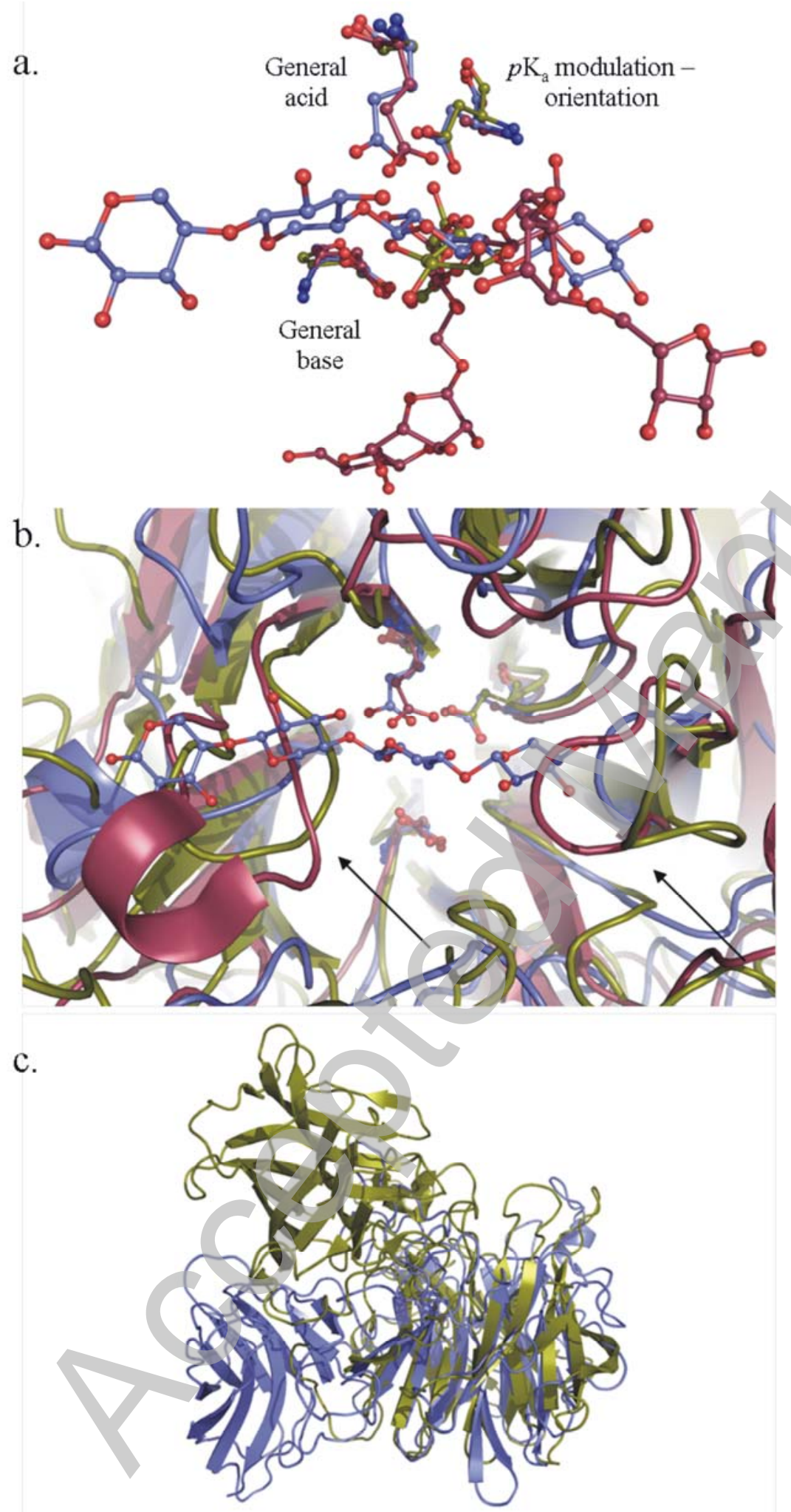

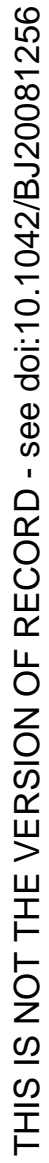

